\title{
Incidence of seizures on continuous EEG monitoring following traumatic brain injury in children
}

\author{
Brent R. O'Neill, MD, ${ }^{1}$ Michael H. Handler, MD, ${ }^{1}$ Suhong Tong, $M S,{ }^{3}$ and Kevin E. Chapman, MD2,3 \\ Departments of ${ }^{1}$ Neurosurgery, ${ }^{2}$ Neurology, and ${ }^{3}$ Pediatrics, University of Colorado, Children's Hospital Colorado, Aurora, \\ Colorado
}

OBJECT Seizures may cause diagnostic confusion and be a source of metabolic stress after traumatic brain injury (TBI) in children. The incidence of electroencephalography (EEG)-confirmed seizures and of subclinical seizures in the pediatric population with $\mathrm{TBI}$ is not well known.

METHODS A routine protocol for continuous EEG (cEEG) monitoring was initiated for all patients with moderate or severe TBI at a Level 1 pediatric trauma center. Over a 3.5-year period, all patients with TBI who underwent cEEG monitoring, both according to protocol and those with mild head injuries who underwent cEEG monitoring at the discretion of the treating team, were identified prospectively. Clinical data were collected and analyzed.

RESULTS Over the study period, 594 children were admitted with TBI, and 144 of these children underwent cEEG monitoring. One hundred two (71\%) of these 144 children had moderate or severe TBI. Abusive head trauma (AHT) was the most common mechanism of injury (65 patients, $45 \%$ ) in children with cEEG monitoring. Seizures were identified on cEEG in 43 patients (30\%). Forty (93\%) of these 43 patients had subclinical seizures, including $17(40 \%)$ with only subclinical seizures and 23 (53\%) with both clinical and subclinical seizures. Fifty-three percent of patients with seizures experienced status epilepticus. Age less than 2.4 years and AHT mechanism were strongly correlated with presence of seizures (odds ratios 8.7 and 6.0, respectively). Those patients with only subclinical seizures had the same risk factors as the other groups. The presence of seizures did not correlate with discharge disposition but was correlated with longer hospital stay and intensive care unit stay.

CONCLUSIONS Continuous EEG monitoring identifies a significant number of subclinical seizures acutely after TBI. Children younger than 2.4 years of age and victims of AHT are particularly vulnerable to subclinical seizures, and seizures in general. Continuous EEG monitoring allows for accurate diagnosis and timely treatment of posttraumatic seizures, and may mitigate secondary injury to the traumatized brain.

http://thejns.org/doi/abs/10.3171/2014.12.PEDS14263

KEY WORDS continuous EEG; traumatic brain injury; abusive head trauma; subclinical seizure; early posttraumatic seizure

$\mathrm{T}$ RAUMATIC brain injury (TBI) is among the most common causes of death and disability in the pediatric population, accounting for 35,000 hospitalizations and more than 2100 deaths annually among children 0-14 years old in the US. ${ }^{7}$ The acute care of patients with severe TBI focuses on the prevention of secondary brain injury from intracranial hypertension, hypoxia, ischemia, elevated intracranial pressure, and other sources. ${ }^{14}$

Seizures can inflict secondary injury to the traumatized brain by imposing an additional metabolic burden and exacerbating other sources of secondary injury. ${ }^{21}$ Seizure activity has been shown in humans to increase intracranial pressure and worsen brain lactate/pyruvate ratios as measured by microdialysis. ${ }^{27}$ Additionally, seizures result in indiscriminant release of neurotransmitters, altering local physiology in potentially harmful ways. ${ }^{27}$

Seizures in the acute post-TBI setting may also cause diagnostic confusion, particularly when the seizures are not witnessed or not recognized, as is common in the case of subclinical seizure. The postictal state commonly alters the neurological examination in unpredictable ways. This may lead to additional treatment, and a diagnostic workup that would be quite different and potentially unnecessary if the seizures had been properly diagnosed. This prob-

ABBREVIATIONS AHT = abusive head trauma; cEEG = continuous EEG; EEG = electroencephalography; GCS = Glasgow Coma Scale; ICU = intensive care unit; ROC = receiver operating characteristic; $\mathrm{TBI}=$ traumatic brain injury.

SUBMITTED June 2, 2014. ACCEPTED December 31, 2014.

INCLUDE WHEN CITING Published online May 8, 2015; DOI: 10.3171/2014.12.PEDS14263.

DISCLOSURE The authors report no conflict of interest concerning the materials or methods used in this study or the findings specified in this paper. 
lem is amplified in pediatric patients because they are more prone to subclinical seizures than adult patients with TBI. ${ }^{2,6,13,15,17,19}$ Continuous electroencephalography (cEEG) is the only test to definitively diagnose subclinical seizures. It additionally offers a continuous record of any seizure activity that may have otherwise gone unwitnessed.

The rate of seizures identified on cEEG in pediatric patients with TBI has not been well studied. We sought to identify the rate of seizures observed when a standard protocol for cEEG in patients with TBI is used, as well as the risk factors for seizures. Such data could provide an assessment of the value of cEEG and guide its utilization. Particular attention is paid to subclinical seizures.

\section{Methods}

\section{Continuous EEG Protocol for Moderate and Severe TBI}

In August 2009 a protocol was implemented at Children's Hospital Colorado calling for 48 hours of cEEG monitoring in all patients with moderate and severe TBI. This treatment protocol was initiated after the occurrence of cases of subclinical seizures in children in which the diagnosis was delayed. Some of these cases presented with decline or fluctuation in neurological status, which after other workups (such as imaging, serum electrolytes, etc.) was evaluated with EEG and diagnosed as subclinical seizure. In other cases, the neurological status was poorer than anticipated from the imaging findings as a result of recurrent subclinical seizure activity.

Children's Hospital Colorado is a Level I pediatric trauma center that serves as a referral center for a large geographic area. TBI is treated at our institution with a multidisciplinary approach involving trauma surgeons, intensive care specialists, neurosurgeons, and other specialists when needed. Treatment proceeds according to an institutional protocol for severe TBI that is based on the Brain Trauma Foundation Guidelines. ${ }^{14}$ Our protocol calls for all patients with moderate and severe TBI to receive anticonvulsant prophylaxis in the form of levetiracetam for 7 days. All patients with moderate and severe injuries are scrupulously monitored for serum sodium levels at a minimum of 4 samplings per day (and more frequently if believed appropriate), and aggressively managed to avoid hyponatremia.

Severe TBI is defined in our guidelines as initial postresuscitation Glasgow Coma Scale (GCS) score of 3-8; moderate TBI is defined as a GCS score of 9-12.24 Continuous EEG monitoring was not applied in instances in which care was deemed to be futile, nor when the initial low GCS score quickly resolved and the TBI was reclassified as mild. Such determinations were made by the neurosurgical providers caring for the patient.

In addition to the moderate and severe TBI cases, continuous EEG monitoring was applied in instances of mild TBI (GCS score 13-15) at the discretion of the treating services. This most commonly occurred when clinical seizures were witnessed or when subclinical seizures were suspected.

Continuous EEG monitoring was initiated as soon as possible following TBI and maintained for a minimum of 48 hours. All studies used gold-plated electrodes ar- ranged in a standard international $10-20$ system, digitized on XLTEK (Natus Medical Incorporated), and paired with bedside video monitoring. A board-certified pediatric neurophysiologist interpreted all studies and directed treatment of seizures when indicated. Our routine is to review studies at least twice per day or more often depending on the occurrence of status epilepticus. The presence of seizures was reported to the team caring for the patient, who then instituted therapy based on their own clinical practice with the assistance of pediatric neurology. Our institutional approach is to treat seizures, and particularly status epilepticus, aggressively. The cEEG data provided the caring providers with guidance on therapy, but a standard treatment protocol was not used.

\section{Continuous EEG Database}

All patients undergoing cEEG monitoring after TBI were prospectively identified for inclusion in a database (henceforth referred to as the cEEG database). Data collection and database formation processes were reviewed and approved by the local institutional review board.

The cEEG database was merged with the trauma registry to allow for examination of a wider range of data elements. This study includes all patients entered into the cEEG database between August 2009 and March 2013. Our primary outcome of interest was rate of seizures. All patients with seizures were classified as having clinical seizures only, subclinical seizures only, or both clinical and subclinical seizures. An electrographic seizure was defined as a paroxysmal EEG discharge that evolved in frequency and location. Subclinical seizure was defined as an electrographic seizure with either no clinical manifestation or with a subtle clinical manifestation that would not be noticed as seizure activity by a bedside caregiver but was identified only on focused review of the video (for example, eye opening or random shifting in bed). All cEEG monitoring was performed with an event marker for nurses to identify events of concern to help clarify subtle changes that could be missed on bedside video monitoring.

The presence of status epilepticus was defined by continuous seizure activity for more than 30 minutes or multiple seizures exceeding $50 \%$ of the EEG recording.

\section{Outcome Variables}

Input variables collected include age, mechanism of injury, presence of witnessed seizure prior to initiation of cEEG, severity of TBI, time to placement of cEEG, and use of prophylactic anticonvulsants. The severity of TBI was assessed by GCS score, intubation status, and number of days in the intensive care unit (ICU). A secondary outcome of interest was the effect of seizures on patient outcome. Information on hospital length of stay and discharge disposition (home, rehabilitation, or death) was collected.

A potential bias was recognized in this data set as some patients underwent cEEG monitoring per the protocol (i.e., for moderate or severe TBI) and some underwent cEEG for witnessed or suspected seizure after mild TBI. These subgroups were analyzed separately. The patients with only subclinical seizures were also analyzed separately. 


\section{Statistical Analysis}

Descriptive statistical analysis was performed to compare patients' input assessment and outcome measures between patients with cEEG monitoring and non-cEEG controls or between seizure and nonseizure patients for the cEEG cohort. The chi-square test was used for binary or categorical data. A t-test or Wilcoxon rank-sum test was used for continuous data. Univariate logistic regressions were performed to model the probability of seizures in relationship to potential predictors. The significant predictors were then selected in a multiple logistic regression model. Receiver operating characteristic (ROC) analysis was used to identify the cutoff of age at injury that best differentiated those who had seizures from those who did not.

\section{Results \\ Patient Population}

Over the 4-year study period, 594 patients were admitted to Children's Hospital Colorado with the diagnosis of TBI. One hundred forty-four of these patients underwent continuous EEG monitoring and were therefore included in the cEEG database (Table 1). Among the 144 in the database, $102(71 \%)$ had moderate or severe brain injury as defined by initial postresuscitation GCS score of 3-12. Thirty-six patients (25\%) had mild brain injury, and $6(4 \%)$ did not have an adequately recorded initial GCS score. Of the 450 trauma patients who did not undergo cEEG, the majority $(321,71 \%)$ had mild brain injury; 14 (3\%) did not have a GCS score recorded, while $27(6 \%)$ had moderate and $88(20 \%)$ had severe TBI based on initial GCS score criteria.

The cEEG population appeared more severely injured based on other parameters as well. Ninety-two percent of cEEG-monitored patients were admitted to the ICU with a mean length of stay of 8.3 days, compared with a $52 \%$ ICU admission rate in the non-cEEG population, and mean ICU stay of 1.5 days $(\mathrm{p}<0.0001)$. Discharge disposition was likewise worse in the cEEG group, with a slight majority of patients discharged to an inpatient rehabilitation service $(67,47 \%)$ or dead $(12,8 \%)$. In comparison, a small minority of the non-cEEG group required inpatient rehabilitation $(23,5 \%)$ and the rate of death was half of the other group $(18,4 \%)$. The population that underwent cEEG was significantly younger and more likely to have abusive head trauma (AHT) as the mechanism of injury than the population that did not undergo cEEG monitoring (Table 1).

\section{Patients in the cEEG Database}

The mean age of those in the cEEG database was 4.3 years. The mean GCS score was 7.7. The most common mechanism of injury was AHT, accounting for 65 patients $(45 \%)$. Other common mechanisms of injury include motor vehicle collisions $(30,21 \%)$, falls $(12,8 \%)$, auto-pedestrian accidents $(9,6 \%)$, crush injury from a heavy object $(9,6 \%)$, sports injuries (primarily skiing; $7,5 \%)$, horse injuries $(6,4 \%)$, and bike accidents $(6,4 \%)$.

Our institutional TBI treatment protocol stipulates anticonvulsants to be administered for 7 days to all moder-
TABLE 1. Injury characteristics of patients with TBI

\begin{tabular}{lccc}
\hline & \multicolumn{2}{c}{ cEEG Monitoring } & \\
\cline { 2 - 3 } Characteristic & Yes $(\mathrm{n}=144)$ & No $(\mathrm{n}=450)$ & p Value \\
\hline $\begin{array}{l}\text { Mean age at injury in yrs } \\
\text { (median) }\end{array}$ & $4.27(4.93)$ & $6.88(5.26)$ & $<0.0001$ \\
\cline { 1 - 2 } Injury mechanism (\%) & & & \\
\hline AHT & $65(45)$ & $59(13)$ & $<0.0001$ \\
\hline All others & $79(55)$ & $391(87)$ & \\
\hline ICU stay (\%) & & & $<0.0001$ \\
\hline Yes & $131(92)$ & $234(52)$ & \\
\hline No & $11(8)$ & $216(48)$ & \\
\hline Mean days in ICU (range) & $8.3(0-32)$ & $1.5(0-19)$ & $<0.0001$ \\
\hline GCS score (\%) & & & $<0.0001$ \\
\hline Mild & $36(25)$ & $321(71)$ & \\
\hline Moderate & $14(10)$ & $27(6)$ & \\
\hline Severe & $88(61)$ & $88(20)$ & \\
\hline Not recorded & $6(4)$ & $14(3)$ & \\
\hline Discharge location (\%) & & & $<0.0001$ \\
\hline Death & $12(8)$ & $18(4)$ & \\
\hline Rehabilitation & $67(47)$ & $23(5)$ & \\
\hline Home & $65(45)$ & $409(91)$ & \\
\hline
\end{tabular}

ate and severely injured patients (initial GCS score 3-12). Ninety-three percent $(95 / 102)$ of such patients in the cEEG database received a prophylactic anticonvulsant. In 75\%, the first dose was given prior to cEEG placement. Levetiracetam was used in $82 \%$ (78 patients) of those receiving a prophylactic anticonvulsant while the remaining 18\% (17 patients) received phenytoin or fosphenytoin. Among the 7 patients who did not get a prophylactic anticonvulsant, 1 had seizures while receiving cEEG, at which point levetiracetam was administered.

\section{Seizure Outcome}

Overall, 43 patients (30\%) had seizures identified on cEEG monitoring, with $93 \%$ of those having at least 1 subclinical seizure. Seventeen patients ( $40 \%$ of those with seizures) had only subclinical seizures while $23(53 \%)$ had both clinical and subclinical seizures. Three patients (7\%) had only clinical seizures. More than half of the patients with seizures $(53 \%, 23$ patients) had status epilepticus (Table 2).

The interval from cEEG placement to first seizure was quite variable, ranging from 2 minutes to more than 3 days. Fifty-one percent of patients who had seizures had their first seizure within 12 hours of cEEG placement, with a median time of 10 hours and 30 minutes. The timing from injury to arrival at Children's Hospital was not always available, particularly in the case of AHT in which the injuries may be multiple and not fully disclosed in the patient's history. For those cases in which the injury could be timed, a mean of 4 hours 19 minutes passed between injury and arrival at Children's Hospital Colorado. A number of these patients were evaluated and stabilized at local hospitals prior to transfer to our institution. Continuous EEG placement occurred at a median of 6 hours after 
TABLE 2. Comparison of patients with and without seizures on cEEG*

\begin{tabular}{|c|c|c|c|}
\hline & \multicolumn{2}{|c|}{ Seizures on cEEG } & \multirow[b]{2}{*}{$\mathrm{p}$ Value } \\
\hline & Yes & No & \\
\hline No. of patients (\%) & $43(30)$ & $101(70)$ & \\
\hline \multicolumn{4}{|l|}{ Seizure type (\%) } \\
\hline Clinical only & $3(7)$ & & \\
\hline Subclinical only & $17(40)$ & & \\
\hline Both & $23(53)$ & & \\
\hline Status epilepticus (\%) & $23(53)$ & & \\
\hline Mean age at injury (yrs) & 1.96 & 5.28 & $<0.0001$ \\
\hline Age $<2.4$ yrs $(\%)$ & & & $<0.0001$ \\
\hline Yes & $37(86)$ & $42(42)$ & \\
\hline No & $6(14)$ & $59(58)$ & \\
\hline Injury mechanism (\%) & & & $<0.0001$ \\
\hline Abusive & $32(74)$ & $33(33)$ & \\
\hline All others & $11(26)$ & $68(67)$ & \\
\hline Initial GCS score (\%) & & & 0.897 \\
\hline $13-15$ & $11(26)$ & $25(26)$ & \\
\hline $9-12$ & $5(12)$ & $9(9)$ & \\
\hline $3-8$ & $26(62)$ & $62(65)$ & \\
\hline Intubation (\%) & & & 0.816 \\
\hline Yes & $5(12)$ & $10(10)$ & \\
\hline No & $38(88)$ & $87(90)$ & \\
\hline ICU admission (\%) & & & 0.5044 \\
\hline Yes & $41(95)$ & $90(91)$ & \\
\hline No & $2(5)$ & $9(9)$ & \\
\hline Median days in ICU (range) & $9(0-24)$ & $5(0-32)$ & 0.0113 \\
\hline $\begin{array}{l}\text { Mean days in hospital } \\
\text { (range) }\end{array}$ & $13.9(2-54)$ & $9.9(1-32)$ & 0.0016 \\
\hline Discharge location (\%) & & & 0.5601 \\
\hline Death & $3(7)$ & $9(9)$ & \\
\hline Rehabilitation & $23(53)$ & $44(44)$ & \\
\hline Home & $17(40)$ & $48(47)$ & \\
\hline
\end{tabular}

${ }^{*}$ Boldface type indicates statistically significant values.

injury. Commencement of cEEG was frequently delayed due to clinical constraints such as the need for repeat cranial imaging or for surgical intervention.

Predictors of seizures were sought by univariate analysis with age, injury mechanism (AHT vs accident), need for intubation, ICU admission, and initial GCS score as input characteristics (Table 3). Young age and AHT mechanism were both strong predictors of seizure (odds ratios [ORs] 8.7 and 6.0, respectively; $\mathrm{p}<0.0001$ for both). Analysis of the ROC curves showed the greatest fidelity when dichotomizing the cohort by age at 2.4 years. None of the remaining factors had a significant effect on the likelihood of seizures. A multiple regression analysis was subsequently performed with age less than 2.4 years and AHT mechanism as input variables. Age was found to be the more powerful predictor of seizures (OR 4.8, $\mathrm{p}=0.01$ ), whereas AHT mechanism did not reach statistical significance in this model (OR 2.3, $\mathrm{p}=0.12)$.
TABLE 3. Univariate and multivariate regression analysis of seizure predictors*

\begin{tabular}{lllr}
\hline \multicolumn{1}{c}{ Analysis } & OR & $95 \% \mathrm{Cl}$ & $\mathrm{p} \mathrm{Value}$ \\
\hline Univariate & & & \\
\hline Age $<2.4$ yrs & 8.7 & $3.4-22.4$ & $<0.0001$ \\
\hline AHT & 6.0 & $2.7-13.6$ & $<0.0001$ \\
\hline Intubation & 1.1 & $0.4-3.6$ & 0.8161 \\
\hline ICU stay & 2.1 & $0.4-9.9$ & 0.3720 \\
\hline Initial GCS score & 1.1 & $0.9-1.3$ & 0.5190 \\
\hline Multivariate regression & & & \\
\hline Age <2.4 yrs & 4.8 & $1.5-15.6$ & 0.0101 \\
\hline AHT & 2.3 & $0.8-6.3$ & 0.1208 \\
\hline
\end{tabular}

* Boldface type indicates statistically significant values.

The patients with only subclinical seizures were compared with the remainder of the seizure group (Table 4). The two groups were very similar. The subclinical seizure-only group trended toward older age ( 2.2 vs 1.8 years old, respectively), accidental injury (65\% of the subclinical seizure group vs $81 \%$ in the other groups), and longer length of hospitalization. None of these differences were statistically significant.

\section{Comparison by Mechanisms of Trauma}

The mean age of those suffering AHT was 0.75 years, whereas those with accidental injuries averaged 7.5 years of age. Only 4 of the AHT cases were more than 2.4 years old, accounting for $6 \%$ of the older cohort. None of these patients experienced seizures. Sixty-one patients of the younger cohort (77\%) were victims of abuse. Those with accidental injuries were on average more severely injured, with a mean GCS score of 6 and 99\% ICU admission, compared with a mean GCS score of 9.7 in the AHT group with $85 \%$ requiring ICU stay. Despite this, seizures were more common in the AHT group. Eleven patients (14\%) in the accidental TBI group had seizures, with $3(4 \%)$ having status epilepticus, whereas 32 patients $(49 \%)$ in the AHT group had seizures, with $20(31 \%)$ experiencing status epilepticus. All of the AHT group with seizures had subclinical seizures; 22 (34\%) had only subclinical seizures, whereas $43(66 \%)$ had both clinical and subclinical seizures. Among the accidental TBI group, 3 $(30 \%)$ of 10 with seizures had only clinical seizures. One patient had both clinical and subclinical seizures while the remainder (6 patients) had subclinical seizures only.

\section{Comparison by Age}

Dividing the cEEG database by age greater or less than 2.4 years yielded similar results to the division by mechanism of injury, as the vast majority of the AHT victims (94\%) were in the younger cohort (Table 5). With this division, the older patients had more severe injuries initially (mean GCS score of 5.5 for the older cohort, 9.6 for the younger cohort). Four patients $(6 \%)$ in the older cohort had a mild brain injury compared with $43 \%$ in the younger cohort. The increased severity of injury in the older children was reflected in the increased likelihood 
TABLE 4. Comparison of patients with subclinical seizures only to patients with any clinical seizures

\begin{tabular}{|c|c|c|c|}
\hline \multirow{2}{*}{ Variables } & \multicolumn{2}{|c|}{ Seizure Type } & \multirow[b]{2}{*}{$\mathrm{p}$ Value } \\
\hline & $\begin{array}{l}\text { Subclinical } \\
\text { Only }\end{array}$ & Others* & \\
\hline Mean age in yrs (median) & $2.23(4.09)$ & $1.78(3.62)$ & 0.7076 \\
\hline Age $<2.4$ yrs $(\%)$ & & & 0.6665 \\
\hline Yes & $14(82)$ & $23(88)$ & \\
\hline No & $3(18)$ & $3(12)$ & \\
\hline Injury mechanism (\%) & & & 0.2379 \\
\hline AHT & $11(65)$ & $21(81)$ & \\
\hline All others & $6(35)$ & $5(19)$ & \\
\hline Intubation (\%) & & & 1.0000 \\
\hline Yes & $2(12)$ & $2(12)$ & \\
\hline No & $15(88)$ & $23(88)$ & \\
\hline ICU stay (\%) & & & 1.0000 \\
\hline Yes & $16(94)$ & $25(96)$ & \\
\hline No & $1(6)$ & $1(4)$ & \\
\hline Median days in ICU (range) & $12(0-24)$ & $7(0-32)$ & 0.2686 \\
\hline Initial GCS score (\%) & & & 0.7334 \\
\hline $13-15$ & $4(23)$ & $7(28)$ & \\
\hline $9-12$ & $3(18)$ & $2(8)$ & \\
\hline $3-8$ & $10(59)$ & $16(64)$ & \\
\hline $\begin{array}{l}\text { Median length of stay in } \\
\text { days (range) }\end{array}$ & $16(5-32)$ & $11.5(2-54)$ & 0.1860 \\
\hline Discharge location (\%) & & & 0.1324 \\
\hline Death & $2(12)$ & $1(4)$ & \\
\hline Rehabilitation & $6(35)$ & $17(65)$ & \\
\hline Home & $9(53)$ & $8(31)$ & \\
\hline Status epilepticus (\%) & $15(58)$ & & 0.7590 \\
\hline
\end{tabular}

* Includes patients with clinical seizures only and patients with both subclinical and clinical seizures.

of intubation and of ICU admission. The younger group was significantly more likely to have seizures than the older group (47\% vs 9\%, respectively; $p<0.0001$ ). All of the younger patients had subclinical seizures, with $38 \%$ of those with seizures having only subclinical seizures. This was significantly different from the pattern in older patients, in which half (3 of 6) had only clinical seizures and half only subclinical. The younger cohort was also more likely to have status epilepticus $(\mathrm{p}<0.0001)$. Status epilepticus occurred in 22 of the younger patients $(59 \%$ of those with seizures, $28 \%$ of the total cohort) and in only 1 of the older patients (17\% of those with seizures, $2 \%$ of the total cohort).

In looking closer at the 6 older patients who had seizures, 3 patients ( 2 with clinical seizures, 1 subclinical) had a very low seizure burden. One had a single clinical seizure lasting less than 1 minute, 1 had a single subclinical seizure lasting 11 minutes, and 1 had only 2 myoclonic seizures lasting a few seconds. One of the 3 patients with more than 2 seizures had a history of epilepsy predating his trauma.
TABLE 5. Comparison by patient age at the time of injury*

\begin{tabular}{|c|c|c|c|}
\hline Characteristic & Age $<2.4$ Yrs & Age $\geq 2.4$ Yrs & p Value \\
\hline No. of patients & 79 & 65 & \\
\hline Seizures & $37(47 \%)$ & $6(9 \%)$ & $<0.0001$ \\
\hline \multicolumn{4}{|l|}{ Seizure type (\%) } \\
\hline Clinical only & $0(0)$ & $3(5)$ & 0.0896 \\
\hline Subclinical only & $14(18)$ & $3(5)$ & 0.0188 \\
\hline Both & $23(29)$ & $0(0)$ & $<0.0001$ \\
\hline Status epilepticus (\%) & $22(28)$ & $1(2)$ & \\
\hline Injury mechanism (\%) & & & $<0.0001$ \\
\hline AHT & $61(77)$ & $4(6)$ & \\
\hline All others & $18(23)$ & $61(94)$ & \\
\hline Mean GCS score & 9.6 & 5.5 & $<0.0001$ \\
\hline Initial GCS score (\%) & & & $<0.0001$ \\
\hline $13-15$ & $32(43)$ & $4(6)$ & \\
\hline $9-12$ & $7(9)$ & $7(11)$ & \\
\hline $3-8$ & $36(48)$ & $52(83)$ & \\
\hline Intubation (\%) & & & 0.0247 \\
\hline Yes & $4(5)$ & $11(18)$ & \\
\hline No & $75(95)$ & $50(82)$ & \\
\hline ICU admission (\%) & & & 0.0230 \\
\hline Yes & $69(87)$ & $62(98)$ & \\
\hline No & $10(13)$ & $1(2)$ & \\
\hline $\begin{array}{l}\text { Median days in ICU } \\
\text { (range) }\end{array}$ & $5(0-29)$ & $8(0-32)$ & 0.0485 \\
\hline $\begin{array}{l}\text { Mean days in hospital } \\
\text { (range) }\end{array}$ & $11.4(2-54)$ & $10.7(1-31)$ & 0.6093 \\
\hline Discharge location (\%) & & & 0.0184 \\
\hline Death & $6(8)$ & $6(9)$ & \\
\hline Rehabilitation & $29(37)$ & $38(58)$ & \\
\hline Home & $44(55)$ & $21(32)$ & \\
\hline
\end{tabular}

* Boldface type indicates statistically significant values.

\section{Comparison by Injury Severity}

Seventy-one percent of this cohort (102 patients) had moderate or severe TBI (initial GCS score of 3-12) and therefore underwent cEEG monitoring according to the protocol, whereas 25\% (36 patients) had mild TBI (GCS score of 13-15). In 4\% the initial GCS score was not recorded in the database (Table 1). There was no significant difference in the rate of seizures captured on cEEG. Twenty-seven percent of the moderate/severe TBI group (28 patients) had seizures compared with $31 \%$ (11 patients) with seizures in the mild TBI group. There were significant differences in the mechanism of injury between the two groups.

The majority of accidental trauma victims $(72,91 \%)$ had moderate or severe brain injuries (GCS score of 3-12) and therefore underwent cEEG according to the protocol. In contrast, $45 \%$ of AHT victims who underwent cEEG (29 patients) had mild brain injury according to initial GCS score (13-15) and were most likely monitored with cEEG for suspicion of seizures by the care team, a wit- 
nessed clinical seizure, or history concerning for seizure. When controlling for injury severity, AHT remained a statistically significant predictor of seizures. Among those patients with moderate or severe TBI, 18 of the AHT group (49\%) had seizures compared with 10 (14\%) of the accidental TBI group $(\mathrm{p}<0.0001)$. Among all patients with mild head injury, $31 \%$ had seizures (11 patients) while on cEEG. All were victims of AHT.

\section{Outcome Predictors}

Among the entire study population, 12 patients (8\%) died during their hospitalization, $67(47 \%)$ were transferred to inpatient rehabilitation, and $65(45 \%)$ were discharged home. This measure of final outcome had no correlation with the presence or absence of seizure (Table 2). There was additionally no correlation with presence of status epilepticus. Twelve patients with status epilepticus (52\%) were transferred to inpatient rehabilitation and 11 (48\%) were discharged home.

Patients with seizures did have a significantly longer hospitalization and longer ICU stay. Seizure patients averaged 13.9 days in the hospital, compared with 9.9 days in those without seizure $(\mathrm{p}=0.002)$. Those with seizures spent a mean of 9.8 days (median 9 days) in the ICU compared with 7.7 days (median 5 days) in those without seizures $(p=0.011)$. Status epilepticus did not correlate with longer hospitalization when compared with seizure patients without status epilepticus (median 7 days vs 10 days, respectively).

\section{Illustrative Cases Case 1}

A 3-month-old girl was transferred to the emergency department from her pediatrician's office with concern for meningitis. The presenting symptoms included lethargy, poor feeding, vomiting, and fussiness. En route to the emergency department, the child had an episode of bradycardia to 85 beats per minute. On arrival the child was lethargic and weakly crying with a bulging fontanel. Her GCS score was 14. A lumbar puncture was performed, which was believed to be traumatic and notable only for blood (352,000 red blood cells). A few hours after presentation to the emergency department, the child became transiently unresponsive with bradycardia and hypertension. She was intubated and a CT scan showed bilateral hypodense subdural fluid collections with a few punctate subarachnoid hemorrhages, raising the suspicion of AHT (Fig. 1). The child's father later admitted to shaking her.

An angiocatheter was placed through the fontanel to drain the subdural fluid and relieve the intracranial pressure. Within a few hours of the subdural drain placement, levetiracetam $(20 \mathrm{mg} / \mathrm{kg} / \mathrm{day})$ was administered and the cEEG monitoring was begun.

The first minutes of the cEEG showed a diffusely slow background. Thirty-two minutes into the recording, the patient began having electrographic seizures with no clinical correlate. Twenty seizures were recorded in the ensuing hour, each lasting from 40 seconds to 2 minutes. Frequent seizures continued for the next 8 hours despite aggressive treatment. By the time seizures were stopped, the patient had received 9 bolus doses of midazolam, a loading dose of fosphenytoin, tripling of the levetiracetam dose, and was on a midazolam drip. Upon review of the video, some seizures were associated with lip smacking and possibly deviation of the eyes (classified as clinical seizures), whereas others had no clinical correlate.

Over the next days, the patient remained seizure free while the midazolam drip was weaned. She was extubated 5 days after admission and discharged after a week of inpatient rehabilitation. She remained on levetiracetam for 1 year after the injury without further seizures. On followup 19 months later, she showed normal development and remained without seizures.

\section{Case 2}

A 16-month-old girl was admitted as a trauma alert after a high-speed motor vehicle accident with a tractortrailer. The patient was restrained in a car seat, but ejected from the vehicle. At a local hospital, she was found to be hypothermic and hypoxic with a GCS score of 3 . The child was stabilized, intubated, and loaded with fosphenytoin prior to transport to our facility. Phenytoin was continued prophylactically. Upon arrival at our facility, the child was moving all limbs spontaneously, localizing pain, and opening eyes to pain. Her GCS score at that time was 8T. A head CT scan showed a right frontal hemorrhagic contusion, diffuse subarachnoid blood over the right convexity, a small subdural hematoma, and several skull fractures. Associated injuries included lung contusions, rib fractures, iliac crest fracture, and liver and spleen lacerations. An intracranial pressure monitor was placed with an initial pressure of $19 \mathrm{~mm} \mathrm{Hg}$. The intracranial pressure remained normal throughout.

Continuous EEG monitoring was begun 7 hours after admission. The initial background was poorly organized with slowing in the delta range and attenuation. There was decreased reactivity in the EEG and occasional spikes over the right posterior temporal area. During this time, the patient was intubated and on vasopressor drips. Drips of midazolam and fentanyl were used for comfort. The first seizure occurred 3 days after admission, lasting for 11 minutes, and involved only the right hemisphere. There was no clinical correlate. Levetiracetam was added. The following day, 3 subclinical seizures occurred, lasting between 10 and 30 minutes. These seizures were recognized rapidly by the electrophysiology team and treated aggressively with an increase in the levetiracetam and phenytoin doses as well as boluses of midazolam. The patient had no further seizures and was weaned off the phenytoin and midazolam. Two and a half years after her injury, the child has not experienced further seizures and is not taking anticonvulsants. She has minimal deficits.

\section{Discussion}

Continuous EEG identified a substantial rate of seizures (30\% of patients) in this cohort of pediatric patients with TBI. Subclinical seizures were very common. Ninety-three percent of those who had seizures (28\% of the total cohort) had at least 1 subclinical seizure and $40 \%$ of those with seizures (12\% of the cohort) had only subclinical seizures. 

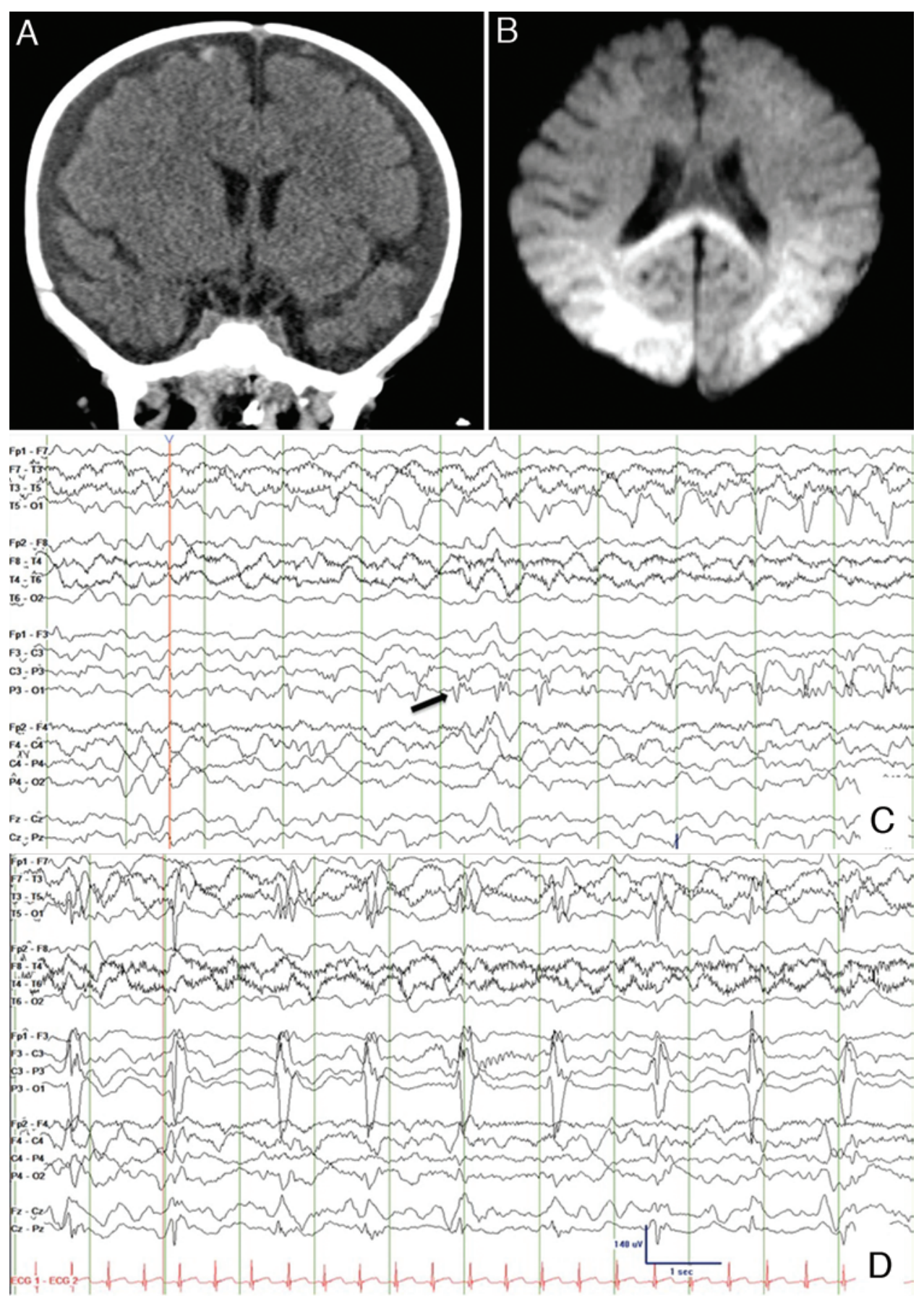

FIG. 1. Images and EEG recordings in a 3-month-old victim of AHT with subclinical seizures. A: Coronal CT scan showing bilateral hypodense subdural collections and punctate subarachnoid hemorrhages near the convexity. B: Diffusion-weighted MR image showing restricted diffusion in the posterior quadrants. C: EEG recordings demonstrating an evolving ictal discharge of about $2 \mathrm{~Hz}$ (arrow) arising from the left occipital region. D: Continuation of the seizure 74 seconds later. There is high-amplitude, $1-\mathrm{Hz}$ spiking reflected in much of the left hemisphere. The total seizure duration was 105 seconds. There was no clinical change on video despite the obvious electrographic discharge. Figure is available in color online only.

Many patients experienced a high seizure burden despite aggressive treatment. Over half of the patients with seizures $(53 \%)$ had status epilepticus. Two factors, young age and AHT mechanism, were strongly predictive of seizures (ORs 8.7 and 6.0, respectively, on univariate analysis). On multiple regression analysis, young age was the stronger predictor of seizures. Markers of severity of trauma, including initial GCS score, need for intubation, and need for ICU admission, did not correlate with seizures. The patients with only subclinical seizures showed no statistical difference from the groups with clinical seizures only or with both types. 
The routine use of prolonged video EEG in pediatric patients with TBI has been explored in 1 other study (which included 27 of the patients in this cohort). ${ }^{2}$ Arndt et al. reviewed 87 pediatric patients with TBI admitted to the ICU and monitored with cEEG for a minimum of 48 hours. They identified seizures in $43 \%$ of their cohort; $38 \%$ of those with seizures had subclinical seizures, and 43\% had status epilepticus (defined as a single seizure $>15$ minutes in duration or $>3$ seizures per hour). The higher overall rate of seizures and lower proportion with subclinical seizures may be the result of their inclusion of impact seizures and other seizures witnessed prior to cEEG placement in the definition of seizure, whereas our study included only seizures captured on cEEG. They also had a smaller proportion of AHT cases (25\%, compared with $45 \%$ in the current study). Similar to our study, Arndt et al. found young age and AHT mechanism to be strongly correlated with seizures, subclinical seizures, and status epilepticus. Severity of injury did not correlate with seizures. $^{2}$

Arango and colleagues prospectively collected clinical and electrographic seizure data on 270 pediatric patients with severe TBI. Continuous EEG was sporadically used. They found an overall seizure rate of $27.7 \%$. Young age and AHT mechanism were identified as independent predictors of seizure. ${ }^{1}$

\section{Younger Patients}

Age proved such a strong predictor of seizures, subclinical seizures, and status epilepticus that it becomes informative to consider younger patients as a separate group. The ROC analysis identified 2.4 years as the most discriminatory cutoff for dividing the groups.

Forty-seven percent of patients in the younger cohort had seizures, with frequent status epilepticus despite being less severely injured than those in the older cohort. All of those with seizures had subclinical seizures, $62 \%$ of whom had only subclinical seizures. Other studies have shown high rates of seizures and subclinical seizures in patients with AHT (Table 6). Hasbani et al. reviewed 32 patients with AHT less than 2 years old, 21 of whom (a more severely injured cohort) underwent cEEG. ${ }^{12}$ Fifty-seven percent of the cEEG group (12 patients) had seizures while monitored. Two-thirds (67\%) of these had only subclinical seizures, and two-thirds had status epilepticus. Seizures were correlated with abnormal EEG background (discontinuous or slow-disorganized) and ischemia on imaging. Clinical seizure at admission did not predict electrographic seizures nor did parenchymal hemorrhage or extraaxial hemorrhage.

Other studies ${ }^{4,5,11}$ have retrospectively assessed seizure frequency in patients with AHT. Continuous EEG was applied sporadically in these cohorts. Bourgeois and colleagues found a seizure frequency of $73 \%$ in 404 patients with AHT. Twenty-five percent had subclinical status epilepticus. ${ }^{5}$ Barlow et al. found an identical rate of seizures (73\%) in 44 patients with AHT, and status epilepticus occurred in $30 \%$ of their cohort. ${ }^{4}$

Abusive head injuries have numerous factors that differentiate them from accidental head injuries and may account for the differences in seizure frequency and type.

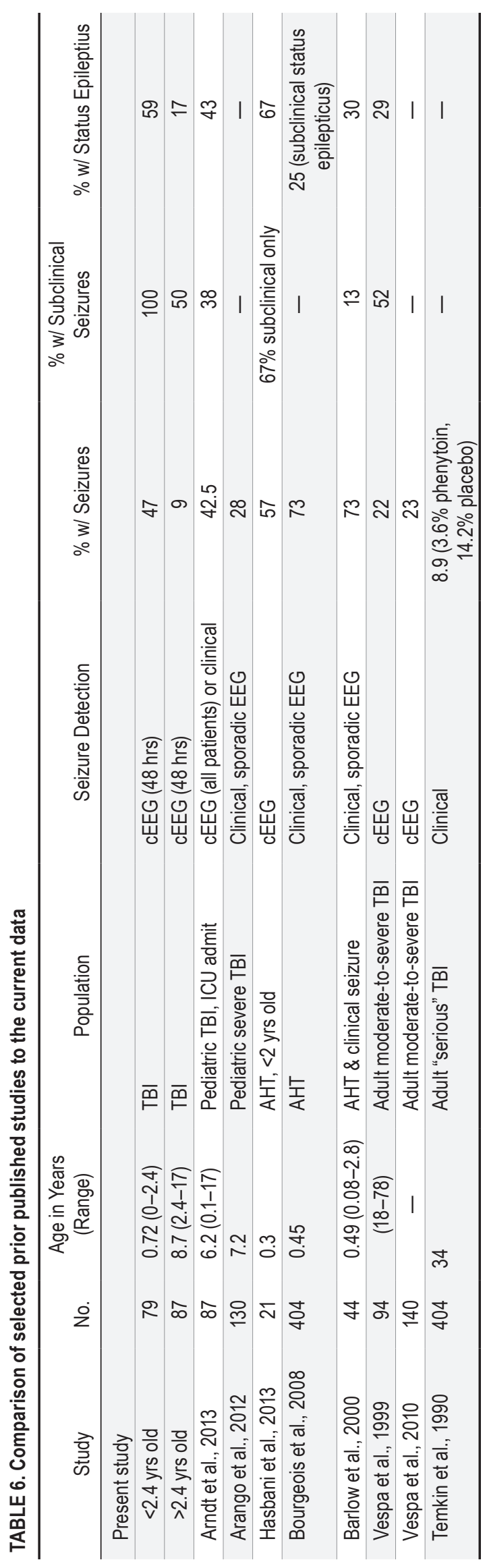


AHT commonly involves a repetitive trauma with multiple episodes of brain injury separated in time prior to the diagnosis of abuse. Such history is often not volunteered by the perpetrator. Even during a single episode of abuse, shaking or repeated impacts may occur, ${ }^{9,10}$ resulting in multiple episodes of acceleration-deceleration of the brain as opposed to the single impact typical of accidental trauma.

Diffusion restriction on MRI is noted in many AHT victims, possibly reflecting a degree of hypoxic-ischemic injury. ${ }^{3,8,20,22,23}$ The proximate cause may be apnea, which is a presenting feature in a significant portion of AHT victims. ${ }^{916}$ Autopsy study of AHT victims has shown a high rate of supratentorial hypoxic brain damage (77\%), while TBI is microscopically evidenced only in the lower medulla and upper cervical spinal cord. ${ }^{9,10}$ Cervicomedullary junction injury may explain the apnea and some part of the supratentorial hypoxic-ischemic injury.

Another major difference in AHT compared with accidental mechanisms is the typical age at injury. In our series, patients with AHT had a mean age of 8 months with only 3 patients (4\%) older than 2.4 years, compared with a mean age of 7.5 years in the accident cohort with $80 \%$ of patients older than 2.4 years. The infant brain is structurally and biochemically different from the brain of an older child. On a macroscopic level, there is significantly less myelination and more water content ${ }^{29}$ in the infant brain, which may predispose them to different types of injury. On a microscopic level, there is less neuronal connectivity and different membrane receptors compared with the brain of the older child. ${ }^{18}$

The intrinsic differences in the brains of infants may account for some degree of the greater propensity for seizures. This hypothesis is supported in our data by the stronger predictive value of young age compared with mechanism of trauma in both the univariate and multivariate analysis. It is also supported by the seizure rate of the young patients $(<2.4$ years old $)$ in the accidental cohort, whose seizure rate of $27 \%$ is nearly midway between the older accident cohort (10\%) and the AHT cohort (49\%).

\section{Older Patients}

Older age was protective in this study, as it has been in many other studies of posttraumatic seizures in pediatric populations. ${ }^{2,6,13,15,17,19}$ Half of our older cohort with seizures had only clinical seizures, which could presumably be recognized without cEEG, whereas only $5 \%$ of the group had any subclinical seizures. The older group also had a lower seizure burden as evidenced by the low rate of status epilepticus and the fact that half of these patients had 1 or 2 seizures. This group more closely resembles an adult cohort than our cohort of infants and toddlers.

In the only prospective adult series of routine cEEG in severe TBI, Vespa et al. found an overall seizure rate of $22 \%$ (identified either clinically or while on cEEG), with $52 \%$ of patients having only subclinical seizures. ${ }^{28}$ Studies that rely on clinical identification of early posttraumatic seizures have found rates ranging from $4 \%$ to $25 \%$ in nonpenetrating injuries. ${ }^{25,30}$ In older patients, cEEG adds less value than in younger patients. A protocol for routine application may not be appropriate in a setting of limited resources.

\section{Assessing the Clinical Value of cEEG}

This observational study reports on the cEEG findings of 144 pediatric patients with TBI in whom cEEG was monitored for 48 hours per our protocol. There was a high incidence of seizures in our study population, including a high rate of subclinical seizures. While it remains to be proven whether detecting and treating seizures improves outcome, cEEG was used clinically to help guide anticonvulsant therapy once seizures were identified. In some ways, the pendulum of cEEG utilization has shifted so that we have found it challenging to discontinue cEEG due to our reassurance that the patient is not having seizures.

While the literature contains no evidence that the routine use of cEEG changes outcome, there are several potential benefits to cEEG. The identification of seizures may prevent diagnostic confusion and eliminate some diagnostic testing when seizures cause a clinical change. Continuous EEG may assist in avoiding overtreatment of unusual nonepileptic events that may be mistaken for seizure activity, such as posturing or autonomic changes. Continuous EEG can also be useful in assessing unexplained persistent alteration of awareness in patients with TBI and may provide some assistance with prognosis. Most importantly, identification of seizures also allows for their treatment, which could potentially mitigate secondary injury to the traumatized brain. Repeated seizures have been associated with an increase in intracranial pressure and alterations of heart rate and blood pressure. Seizures additionally cause significant energy consumption by the brain. The combined effect of these features creates metabolic stress on the injured brain, an effect demonstrated by the alterations in lactate/pyruvate ratio identified with microdialysis. ${ }^{26,27}$ Early recognition of seizures offers an opportunity to intervene in this cascade.

Routine use of cEEG identities a significant number of subclinical seizures, particularly in very young patients with TBI. The value of this monitoring is considerably lower in older children, in whom seizures occur less frequently. The observation that seizures are much more common in young patients with TBI may help guide resource utilization given the scarcity and complexity of cEEG. Our study would support the routine use of cEEG in children younger than 2.4 years of age and in children with AHT to identify seizure activity.

\section{Conclusions}

When routinely applied, cEEG identifies a significant rate of seizures, including frequent subclinical seizures in patients with TBI younger than 2.4 years and in those with abusive head trauma. The considerable risk of subclinical seizures in this patient population should be recognized when treating pediatric TBI. While there is currently no evidence that cEEG alters outcome in pediatric TBI, its routine application does have the potential benefits of mitigating diagnostic confusion and allowing for earlier treatment when seizures are present.

\section{References}

1. Arango JI, Deibert CP, Brown D, Bell M, Dvorchik I, Adelson PD: Posttraumatic seizures in children with severe 
traumatic brain injury. Childs Nerv Syst 28:1925-1929, 2012

2. Arndt DH, Lerner JT, Matsumoto JH, Madikians A, Yudovin $\mathrm{S}$, Valino H, et al: Subclinical early posttraumatic seizures detected by continuous EEG monitoring in a consecutive pediatric cohort. Epilepsia 54:1780-1788, 2013

3. Babikian T, Tong KA, Galloway NR, Freier-Randall MC, Obenaus A, Ashwal S: Diffusion-weighted imaging predicts cognition in pediatric brain injury. Pediatr Neurol 41:406412, 2009

4. Barlow KM, Spowart JJ, Minns RA: Early posttraumatic seizures in non-accidental head injury: relation to outcome. Dev Med Child Neurol 42:591-594, 2000

5. Bourgeois M, Di Rocco F, Garnett M, Charron B, Boddaert $\mathrm{N}$, Soufflet C, et al: Epilepsy associated with shaken baby syndrome. Childs Nerv Syst 24:169-173, 2008

6. Chiaretti A, De Benedictis R, Polidori G, Piastra M, Iannelli A, Di Rocco C: Early post-traumatic seizures in children with head injury. Childs Nerv Syst 16:862-866, 2000

7. Faul M, Xu L, Wald MM, Coronado VG: Traumatic Brain Injury in the United States: Emergency Department Visits, Hospitalizations and Deaths 2002-2006. Atlanta: Centers for Disease Control and Prevention, National Center for Injury Prevention and Control, 2010

8. Galloway NR, Tong KA, Ashwal S, Oyoyo U, Obenaus A: Diffusion-weighted imaging improves outcome prediction in pediatric traumatic brain injury. J Neurotrauma 25:11531162,2008

9. Geddes JF, Hackshaw AK, Vowles GH, Nickols CD, Whitwell HL: Neuropathology of inflicted head injury in children. I. Patterns of brain damage. Brain 124:1290-1298, 2001

10. Geddes JF, Vowles GH, Hackshaw AK, Nickols CD, Scott IS, Whitwell HL: Neuropathology of inflicted head injury in children. II. Microscopic brain injury in infants. Brain 124:1299-1306, 2001

11. Goldstein JL, Leonhardt D, Kmytyuk N, Kim F, Wang D, Wainwright MS: Abnormal neuroimaging is associated with early in-hospital seizures in pediatric abusive head trauma. Neurocrit Care 15:63-69, 2011

12. Hasbani DM, Topjian AA, Friess SH, Kilbaugh TJ, Berg RA, Christian CW, et al: Nonconvulsive electrographic seizures are common in children with abusive head trauma. Pediatr Crit Care Med 14:709-715, 2013

13. Hendrick EB, Harris L: Post-traumatic epilepsy in children. J Trauma 8:547-556, 1968

14. Kochanek PM, Carney N, Adelson PD, Ashwal S, Bell MJ, Bratton S, et al: Guidelines for the acute medical management of severe traumatic brain injury in infants, children, and adolescents-second edition. Pediatr Crit Care Med 13 Suppl 1:S1-S82, 2012

15. Liesemer K, Bratton SL, Zebrack CM, Brockmeyer D, Statler KD: Early post-traumatic seizures in moderate to severe pediatric traumatic brain injury: rates, risk factors, and clinical features. J Neurotrauma 28:755-762, 2011

16. Maguire S, Pickerd N, Farewell D, Mann M, Tempest V, Kemp AM: Which clinical features distinguish inflicted from non-inflicted brain injury? A systematic review. Arch Dis Child 94:860-867, 2009

17. Ong LC, Dhillon MK, Selladurai BM, Maimunah A, Lye MS: Early post-traumatic seizures in children: clinical and radiological aspects of injury. J Paediatr Child Health 32:173-176, 1996
18. Rakhade SN, Jensen FE: Epileptogenesis in the immature brain: emerging mechanisms. Nat Rev Neurol 5:380-391, 2009

19. Ratan SK, Kulshreshtha R, Pandey RM: Predictors of posttraumatic convulsions in head-injured children. Pediatr Neurosurg 30:127-131, 1999

20. Schaefer PW, Huisman TA, Sorensen AG, Gonzalez RG, Schwamm LH: Diffusion-weighted MR imaging in closed head injury: high correlation with initial Glasgow Coma Scale score and score on modified Rankin scale at discharge. Radiology 233:58-66, 2004

21. Schreiber JM, Zelleke T, Gaillard WD, Kaulas H, Dean N, Carpenter JL: Continuous video EEG for patients with acute encephalopathy in a pediatric intensive care unit. Neurocrit Care 17:31-38, 2012

22. Sieswerda-Hoogendoorn T, Boos S, Spivack B, Bilo RAC, van Rijn RR: Abusive head trauma part II: radiological aspects. Eur J Pediatr 171:617-623, 2012

23. Sieswerda-Hoogendoorn T, Boos S, Spivack B, Bilo RAC, van Rijn RR: Educational paper: Abusive head trauma part I. Clinical aspects. Eur J Pediatr 171:415-423, 2012

24. Teasdale G, Jennett B: Assessment of coma and impaired consciousness. A practical scale. Lancet 2:81-84, 1974

25. Temkin NR, Dikmen SS, Wilensky AJ, Keihm J, Chabal S, Winn HR: A randomized, double-blind study of phenytoin for the prevention of post-traumatic seizures. N Engl J Med 323:497-502, 1990

26. Vespa PM, McArthur DL, Xu Y, Eliseo M, Etchepare M, Dinov I, et al: Nonconvulsive seizures after traumatic brain injury are associated with hippocampal atrophy. Neurology 75:792-798, 2010

27. Vespa PM, Miller C, McArthur D, Eliseo M, Etchepare M, Hirt D, et al: Nonconvulsive electrographic seizures after traumatic brain injury result in a delayed, prolonged increase in intracranial pressure and metabolic crisis. Crit Care Med 35:2830-2836, 2007

28. Vespa PM, Nuwer MR, Nenov V, Ronne-Engstrom E, Hovda DA, Bergsneider M, et al: Increased incidence and impact of nonconvulsive and convulsive seizures after traumatic brain injury as detected by continuous electroencephalographic monitoring. J Neurosurg 91:750-760, 1999

29. Welker KM, Patton A: Assessment of normal myelination with magnetic resonance imaging. Semin Neurol 32:15-28, 2012

30. Yablon SA: Posttraumatic seizures. Arch Phys Med Rehabil 74:983-1001, 1993

\section{Author Contributions}

Conception and design: O'Neill, Handler, Chapman. Acquisition of data: O'Neill, Chapman. Analysis and interpretation of data: O'Neill, Handler. Drafting the article: O'Neill. Critically revising the article: Handler, Chapman. Reviewed submitted version of manuscript: O’Neill, Handler, Chapman. Statistical analysis: Tong.

\section{Correspondence}

Brent O'Neill, Department of Neurosurgery, University of Colorado, Children's Hospital Colorado, 13123 E. 16th Ave., B-330, Aurora, CO 80045. email: brent.oneill@childrenscolorado.org. 